\title{
ON EXISTENCE OF SOLUTIONS TO STOCHASTIC DIFFERENTIAL INCLUSIONS WITH CURRENT VELOCITIES II
}

\author{
Yu. E. Gliklikh ${ }^{1}$, yeg@math.vsu.ru, \\ A. V. Makarova ${ }^{1}$, allagm@mail.ru. \\ ${ }^{1}$ Voronezh State University, Voronezh, Russian Federation.
}

\begin{abstract}
Existence of solution theorems are obtained for stochastic differential inclusions given in terms of the so-called current velocities (symmetric mean derivatives, a direct analogs of ordinary velocity of deterministic systems) and quadratic mean derivatives (giving information on the diffusion coefficient) on the flat $n$-dimensional torus. Right-hand sides in both the current velocity part and the quadratic part are set-valued but satisfy some natural conditions, under which they have $\varepsilon$-approximations that point-wise converge to Borel measurable selections of the corresponding set-valued mappings.
\end{abstract}

Keywords: mean derivatives, current velocities, differential inclusions

\section{Introduction}

This paper paper is devoted to the same topic as [8], but here we deal with another type of right-hand sides of the inclusion, both for the part with current velocities and for that with quadratic mean derivatives.

Recall that the notion of mean derivatives was introduced by Edward Nelson [10, 11, 12] for the needs of stochastic mechanics (a version of quantum mechanics). The equation of motion in this theory (called the Newton - Nelson equation) was the first example of equations in mean derivatives. Later it turned out that the equations in mean derivatives arose also in many other branches of science (mechanics, hydrodynamics, Navier - Stokes vortices, gauge fields, economics, etc.).

Nelson introduced forward and backward mean derivatives while only their half-sum, symmetric mean derivative called current velocity, is a direct analog of ordinary velocity for deterministic processes. In [2] another mean derivative called quadratic, is introduced. It gives information on the diffusion coefficient of the process and using Nelson's and quadratic mean derivatives together, one can in principle recover the process from its mean derivatives.

Since the current velocities are natural analogs of ordinary velocities of deterministic processes, investigation of equations and especially inclusions with current velocities is very much important for applications since there are a lot of models of various physical, economical etc. processes based on such equations and inclusions.

In [8] we investigated the inclusions with current velocities in the case where both the part with current velocities and that with quadratic mean derivatives in the right-hand side of the inclusion had smooth selectors. Here we deal with another sort of right-hand side: they satisfy some natural conditions, under which they have $\varepsilon$-approximations that pointwise converge to Borel measurable selections of the corresponding set-valued mappings. 
To avoid some technical difficulties we consider the inclusions on the flat $n$-dimensional torus $\mathcal{T}^{n}$. This means that the torus is considered as a quotient space of $\mathbb{R}^{n}$ relative to the integral lattice and that the Riemannian metric on $\mathcal{T}^{n}$ is inherited from the Euclidean metric in $\mathbb{R}^{n}$. Everywhere below we use the operations of addition and subtraction of points and integration in $\mathcal{T}^{n}$ as in $\mathbb{R}^{n}$ modulo factorization relative to the integral lattice. The construction and notation of stochastic integrals and stochastic differential equations on $\mathcal{T}^{n}$ are the same as in $\mathbb{R}^{n}$ because of the use of Euclidean metric.

The detailed exposition of preliminary notions and facts from the Theory of Mean Derivatives used in the paper, can be found in [7]. For the Theory of Set-Valued Mappings we recommend [3].

Everywhere in the paper we use Einstein's convention of summation relative to a shared upper and lower index (see, e.g., [7]).

\section{Preliminaries on Mean Derivatives}

For the sake of convenience here we have to repeat shortly the preliminaries on mean derivatives from [8]. Consider the $n$-dimensional flat torus $\mathcal{T}^{n}$. We shall deal with stochastic processes in $\mathcal{T}^{n}$ given on a certain probability space $(\Omega, \mathcal{F}, \mathrm{P}), t \in[0, T] \subset \mathbb{R}$.

Denote by $\mathcal{P}_{t}^{\xi}$ the sub- $\sigma$-algebra of $\mathcal{F}$ generated by preimages of Borel sets from $\hbar \mathcal{T}^{n}$ by all mappings $\xi(s): \Omega \rightarrow \mathbb{R}^{n}$ for $0 \leq s \leq t$; $\mathcal{P}_{t}^{\xi}$ is called the "past" for $\xi(t)$.

Denote by $\mathcal{N}_{t}^{\xi}$ the sub- $\sigma$-algebra of $\mathcal{F}$ generated by preimages of Borel sets from $\mathcal{T}^{n}$ by the mapping $\xi(t): \Omega \rightarrow \mathcal{T}^{n} ; \mathcal{N}_{t}^{\xi}$ is called the "present" for $\xi(t)$.

The $\sigma$-subalgebras $\mathcal{P}_{t}^{\xi}$ and $\mathcal{N}_{t}^{\xi}$ for all $t$ are supposed to be complete, i.e., containing all sets of probability zero. Obviously $\mathcal{N}_{t}^{\xi}$ is a sub- $\sigma$-algebra in $\mathcal{P}_{t}^{\xi}$.

For the sake of convenience we denote by $E_{t}^{\xi}$ the conditional expectation $E\left(\cdot \mid \mathcal{N}_{t}^{\xi}\right)$ with respect to $\mathcal{N}_{t}^{\xi}$ for $\xi(t)$.

As in $[10,11,12]$, we introduce the following notions of forward and backward mean derivatives.

Definition 1. (i) The forward mean derivative $D \xi(t)$ of $\xi(t)$ at the time instant $t$ is an $L_{1}$ random element of the form

$$
D \xi(t)=\lim _{\triangle t \rightarrow+0} E_{t}^{\xi}\left(\frac{\xi(t+\triangle t)-\xi(t)}{\triangle t}\right),
$$

where the limit is supposed to exist in $L_{1}(\Omega, \mathcal{F}, \mathrm{P})$ and $\triangle t \rightarrow+0$ means that $\triangle t$ tends to 0 and $\triangle t>0$.

(ii) The backward mean derivative $D_{*} \xi(t)$ of $\xi(t)$ at $t$ is the $L_{1}$-random element

$$
D_{*} \xi(t)=\lim _{\Delta t \rightarrow+0} E_{t}^{\xi}\left(\frac{\xi(t)-\xi(t-\Delta t)}{\Delta t}\right)
$$

where (as well as in (i)) the limit is assumed to exist in $L^{1}(\Omega, \mathcal{F}, \mathrm{P})$ and $\Delta t \rightarrow+0$ means that $\Delta t \rightarrow 0$ and $\Delta t>0$.

As usual in the machinery of conditional expectation (see, e.g., [13]), there exist Borel measurable vector fields $a^{\xi}(t, m)$ and $a_{*}^{\xi}(t, m)$ such that $D \xi(t)=a^{\xi}(t, \xi(t))$ and $D_{*} \xi(t)=$ $a_{*}^{\xi}(t, \xi(t))$, respectively. 
Definition 2. The derivative $D_{S}=\frac{1}{2}\left(D+D_{*}\right)$ is called the symmetric mean derivative. The derivative $D_{A}=\frac{1}{2}\left(D-D_{*}\right)$ is called the antisymmetric mean derivative.

Consider the vectors $v^{\xi}(t, x)=\frac{1}{2}\left(a^{\xi}(t, x)+a_{*}^{\xi}(t, x)\right)$ and $u^{\xi}(t, x)=\frac{1}{2}\left(a^{\xi}(t, x)-a_{*}^{\xi}(t, x)\right)$.

Definition 3. $v^{\xi}(t)=v^{\xi}(t, \xi(t))=D_{S} \xi(t)$ is called the current velocity of the process $\xi(t)$; $u^{\xi}(t)=u^{\xi}(t, \xi(t))=D_{A} \xi(t)$ is called the osmotic velocity of the process $\xi(t)$.

The physical meaning of current velocity is a direct analog of the ordinary velocity of a deterministic process. The osmotic velocity measures how fast the randomness increases. This interpretation becomes clear from the following features of $v^{\xi}$ and $u^{\xi}$ (see [12]).

Consider an autonomous smooth field of non-degenerate linear operators $A(x): \mathbb{R} \times$ $\mathbb{R}^{n} \rightarrow \mathbb{R}^{n}, x \in \mathcal{T}^{n}$. Suppose that $\xi(t)$ is a diffusion type process whose diffusion integrand is $A(t, \xi(t))$. Then its diffusion coefficient $A(x) A^{*}(x)$ is a smooth field of symmetric positive definite $(2,0)$-tensors with matrices $\alpha(x)=\left(\alpha^{i j}(x)\right)$. Since all those matrices are nondegenerate, the field of inverse matrices $\left(\alpha_{i j}(x)\right)$ exists and is smooth and at any $(x)$ the matrix $\left(\alpha_{i j}\right)(x)$ is symmetric and positive definite. Thus it defines a new Riemannian metric (symmetric positive definite $(0,2)$-tensor field) $\alpha(\cdot, \cdot)=\alpha_{i j} d x^{i} \otimes d x^{j}$ on $\mathcal{T}^{n}$. Consider the Riemannian volume form of this Riemannian metric $\Lambda_{\alpha}=\sqrt{\operatorname{det}\left(\alpha_{i j}\right)} d x^{1} \wedge d x^{2} \wedge \cdots \wedge d x^{n}$.

Denote by $\rho^{\xi}(t, x)$ the probability density of $\xi(t)$ with respect to the volume form $d t \wedge \Lambda_{\alpha}=\sqrt{\operatorname{det}\left(\alpha_{i j}\right)} d t \wedge d x^{1} \wedge d x^{2} \wedge \cdots \wedge d x^{n}$ on $[0, T] \times \mathcal{T}^{n}$, i.e., for any continuous bounded function $f:[0, T] \times \mathcal{T}^{n} \rightarrow \mathbb{R}$ the relation

$$
\int_{0}^{T} E(f(t, \xi(t))) d t=\int_{0}^{T}\left(\int_{\Omega} f(t, \xi(t)) d \mathrm{P}\right) d t=\int_{0}^{T}\left(\int_{\mathbb{R}^{n}} f(t, x) \rho^{\xi}(t, x) \Lambda_{\alpha_{t}}\right) d t
$$

holds. Then under the assumption that $\rho^{\xi}(t, x)$ nowhere equals zero

$$
u^{\xi}(t, x)=\frac{1}{2} \frac{\frac{\partial}{\partial x^{j}}\left(\alpha^{i j}(t, x) \rho^{\xi}(t, x)\right)}{\rho^{\xi}(t, x)} \frac{\partial}{\partial x^{i}}
$$

where $\left(\alpha^{i j}\right)$ is the matrix of operator $A A^{*}$. Formula (4) is proved in [4].

For $v^{\xi}(t, x)$ and $\rho^{\xi}(t, x)$ the so called equation of continuity

$$
\frac{\partial \rho^{\xi}(t, x)}{\partial t}=-\operatorname{Div}\left(v^{\xi}(t, x) \rho^{\xi}(t, x)\right)
$$

holds, where Div denotes divergence with respect to the Riemannian metric $\alpha(\cdot, \cdot)$. Formula (5) is proved in [2].

Following [1] we introduce the differential operator $D_{2}$ that differentiates an $L_{1}$ random process $\xi(t), t \in[0, T]$ according to the rule

$$
D_{2} \xi(t)=\lim _{\triangle t \rightarrow+0} E_{t}^{\xi}\left(\frac{(\xi(t+\triangle t)-\xi(t))(\xi(t+\Delta t)-\xi(t))^{*}}{\triangle t}\right),
$$

where $(\xi(t+\triangle t)-\xi(t))$ is considered as a column vector (vector in $\left.\mathbb{R}^{n}\right),(\xi(t+\triangle t)-\xi(t))^{*}$ is a row vector (transposed, or conjugate vector) and the limit is supposed to exists in $L_{1}(\Omega, \mathcal{F}, \mathrm{P})$. We emphasize that the matrix product of a column on the left and a row on the 
right is a matrix with rank 1 but after passing to limit and taking conditional expectation $D_{2} \xi(t)$ becomes a symmetric semi-positive definite matrix function on $[0, T] \times \mathbb{R}^{n}$ (in many cases positive definite). We call $D_{2}$ the quadratic mean derivative. It takes values in the set of $(2,0)$-tensors having symmetric positive semi-definite matrices.

As mentioned above, the current velocity is analogous to ordinary velocity for a nonrandom process. Thus, from the physical point of view, it is an important problem to study equations and inclusions with current velocities.

Let $v(t, m)$ be a vector field and $\alpha(t, m)$ be a symmetric positive semi-definite $(2,0)$ tensor field on $\mathcal{T}^{n}$. The system

$$
\left\{\begin{array}{l}
D_{S} \xi(t)=v(t, \xi(t)) \\
D_{2} \xi(t)=\alpha(t, \xi(t))
\end{array}\right.
$$

is called the first order differential equation with current velocities.

Note that equation (7) on the flat torus $\mathcal{T}^{n}$ can be considered as an equation on $\mathbb{R}^{n}$ periodic in space variables.

Definition 4. We say that (7) on $\mathcal{T}^{n}$ has a solution on $[0, T]$ with initial condition $\xi(0)=$ $\xi_{0}$ if there exists a probability space $(\Omega, \mathcal{F}, \mathrm{P})$ and a process $\xi(t)$ given on $(\Omega, \mathcal{F}, \mathrm{P})$ and taking values in $\mathcal{T}^{n}$ such that $\xi(0)=\xi_{0}$ and for almost all $t \in[0, T]$ equation (7) is satisfied P-a.s. by $\xi(t)$.

Theorem 1. Let $v:[0, T] \times \mathcal{T}^{n} \rightarrow \mathbb{R}^{n}$ and $\alpha: \mathcal{T}^{n} \rightarrow \mathrm{S}_{+}(n)$ be smooth (so $\alpha$ determines the Riemannian metric $\alpha(\cdot, \cdot)$ on $\mathcal{T}^{n}$, introduced above). Let $\xi_{0}$ be a random element with values in $\mathcal{T}^{n}$ whose probability density $\rho_{0}$ with respect to the volume form $\Lambda_{\alpha}$ of $\alpha(\cdot, \cdot)$ on $\mathcal{T}^{n}$ (see above) is smooth and nowhere equal to zero. Then for the initial condition $\xi(0)=\xi_{0}$ equation $(7)$ has a solution that is well-defined on the entire interval $t \in[0, T]$.

Theorem 1 is a simple corollary to the main result of [2]. Here we use the fact that on the compact manifold $\mathcal{T}^{n}$ the right-hand sides of (7) are uniformly bounded and so the hypothesis of the existence theorem from [2] is fulfilled.

Introduce $p_{0}=\log \rho_{0}$ and consider $p(t, m)=\log \rho^{\xi}(t, m)$ where $\rho^{\xi}(t, m)$ is the density (3) corresponding to the solution $\xi(t)$ of $(7)$. It is shown in [2, Theorem 3] that $p(t, m)$ is well-posed and takes the form

$$
p(t, m)=p_{0}\left(g_{-t}(m)\right)-\int_{0}^{t}(\operatorname{Div} v)\left(s, g_{s}\left(g_{-t}(m)\right) d s\right.
$$

where Div is the divergence with respect to $\alpha(\cdot, \cdot)$ and $g_{t}$ is the flow of smooth vector field $v(t, m)$.

\section{Some Technical Constructions}

Lemma 1. Let $\alpha(x)$ be a jointly continuous mapping from $[0, T] \times \mathbb{R}^{n}$ to $S_{+}(n)$. Then there exists a jointly continuous mapping $A(x)$ from $[0, T] \times \mathbb{R}^{n}$ to the space $L\left(\mathbb{R}^{n}, \mathbb{R}^{n}\right)$ such that for any $x \in \mathbb{R}^{n}$ the equality $A(x) A^{*}(x)=\alpha(x)$ holds.

Proof. Since the symmetric matrices $\alpha(x)$ are positive definite, all their angular minors are positive and in particular do not equals zero. Then the so-called Gauss decomposition 
holds (see [14, Теорема II.9.3]): $\alpha=\zeta \delta z$, where $\zeta$ is a lower-triangle matrix with units along the diagonal, $z$ is an upper-triangle matrix with units along the diagonal and $\delta$ is a diagonal matrix. In addition the elements of $\zeta, \delta$ and $z$ are rationally expressed via the elements of $\alpha$, i.e., those matrices are continuous in $x$. From the fact that $\alpha$ are a symmetric matrices, one can easily see that $z=\zeta^{*}(z$ is the transposed $\zeta)$. One also can easily see that in this situation the elements of diagonal matrix $\delta$ are positive. Hence, the diagonal matrix $\sqrt{\delta}$, in which on the diagonal the square roots of the corresponding elements of $\delta$ are located, is well-posed. Consider the matrices $A(x)=\zeta \sqrt{\delta}$. By the construction $A(x)$ is continuous in $x$ and $A(x) A^{*}(x)=\zeta(x) \delta(x) z(x)=\alpha(x)$.

Corollary 1. If in the hypothesis of Lemma $1 \alpha(x)$ is not continuous but Borel measurable or smooth, there exists Borel measurable or smooth, respectively, mapping $A(x)$ from $\mathbb{R}^{n}$ to the space $L\left(\mathbb{R}^{n}, \mathbb{R}^{n}\right)$ such that for any $x \in \mathbb{R}^{n}$ the equality $A(x) A^{*}(x)=\alpha(x)$ holds.

Denote by $\mathrm{T}_{-}(n)$ the set of lower-triangle $n \times n$ matrices with zeros along the diagonal. It is a linear subspace in the space $\mathbb{R}^{n^{2}}$ of all $n \times n$ matrices. Evidently $\zeta$, introduced above, belongs to the linear sub-manifold $\mathrm{T}_{-}(n)+I$ in $\mathbb{R}^{n^{2}}$, where $I$ is the unit $n \times n$ matrix.Denote by $\mathrm{T}: S_{+}(n) \rightarrow \mathrm{T}_{-}(n)$ the smooth mapping $\alpha \in S_{+}(n)$ to

$$
\mathrm{T} \alpha=\zeta-I \in \mathrm{T}_{-}(n) .
$$

Denote by $S_{L C}$ the set of symmetric positive definite matricas with constant (equal to $C>0$ ) determinant. In particular, this means that $\delta_{1} \cdot \ldots \cdot \delta_{n}=$ const $=C$ and $\sqrt{\delta_{1}} \cdot \ldots \cdot \sqrt{\delta_{n}}=\sqrt{C}$, where the dot denote the multiplication.

Let $\mathrm{L}_{0}(n)$ be a linear subspace in $\mathbb{R}^{n}$ that consists of the vectors $X=\left(X^{1}, \ldots, X^{n}\right)$ such that $X^{1}+\ldots+X^{n}=0$. Introduce the smooth mapping $\mathrm{L}_{C}: S_{L C} \rightarrow \mathrm{L}_{0}$, that sends the symmetric matrix $\alpha \in S_{L C}$ to

$$
\mathrm{L}_{\mathrm{C}}(\alpha)=\left(\ln \frac{\sqrt{\delta_{1}}}{\sqrt{C}}, \ldots, \ln \frac{\sqrt{\delta_{n}}}{\sqrt{C}}\right) \in \mathrm{L}_{0}(n),
$$

where $\delta_{i}$ are diagonal elements of $\delta$ correspondent to $\alpha$.

Note that $\mathrm{T}_{-}(n)$ and $\mathrm{L}_{0}(n)$ are linear spaces, i.e., the notion of convex set in those spaces is well-posed.

Consider a smooth field of symmetric positive definite $(2,0)$-tensors $\alpha(m)=\left(\alpha^{i j}(m)\right)$ on the torus. Since all those matrices are non-degenerated, the field of inverse matrices $\left(\alpha_{i j}\right)$ is well-posed and smooth. In addition at every $m$ the matrix $\left(\alpha_{i j}\right)(m)$ is symmetric and positive definite. Thus the latter field can be considered as a new Riemannian metric on $\mathcal{T}^{n}$ (a smooth field of symmetric positive definite $(0,2)$-tensors) $\alpha(\cdot, \cdot)=\alpha_{i j} d q^{i} \otimes d q^{j}$. Consider its volume form $\Lambda_{\alpha}=\sqrt{\operatorname{det}\left(\alpha_{i j}\right)} d q^{1} \wedge d q^{2} \wedge \cdots \wedge d q^{n}$.

Lemma 2. ([9]) For any smooth autonomous $(2,0)$-tensor field $\alpha(m)$ on the flat torus $\mathcal{T}^{n}$ with the values in $S_{L C}$ :

(i) The volume form $\Lambda_{\alpha}$ of the corresponding Riemannian metric $\alpha(\cdot, \cdot)$ is equal to $\sqrt{C} \Lambda_{E}$, where $\Lambda_{E}=d q^{1} \wedge \cdots \wedge d q^{n}$ is the volume form of the Euclidean metric on $\mathcal{T}^{n}$, inherited from $\mathbb{R}^{n}$ after factorization with respect to integral lattice.

(ii) For any smooth vector field $v(t, m)$ on $\mathcal{T}^{n}$ its divergence $\operatorname{Div} v$ with respect to $\Lambda_{\alpha}$ coincides with the ordinary divergence $\operatorname{div} v$ (i.e. with respect to $\Lambda_{E}$ ). 
(iii) For any random element with values in $\mathcal{T}^{n}$ its density of distribution with respect to $\Lambda_{\alpha}$ equals the density of distribution with respect to $\Lambda_{E}$, divided by $\sqrt{C}$.

Proof. Indeed, Действительно, $\Lambda_{\alpha}=\sqrt{\operatorname{det}\left(\alpha_{i j}\right)} d q^{1} \wedge \cdots \wedge d q^{n}$ and since $\operatorname{det}\left(\alpha_{i j}\right)=C$, $\Lambda_{\alpha}=\sqrt{C} \Lambda_{E}=\sqrt{C} d q^{1} \wedge \cdots \wedge d q^{n}$

Recall that Div $v$ can be found from the equlity

$$
\mathcal{L}_{v} \Lambda_{\alpha}=(\operatorname{Div} v) \Lambda_{\alpha}
$$

where $\mathcal{L}_{v}$ is the Lie derivative along $v$ (see details, e.g., in [7]). Recall also that $\mathcal{L}_{v} \Lambda_{\alpha}=$ $\left.d(v\rfloor \Lambda_{\alpha}\right)$, where $\rfloor$ denotes internal multiplication of vectors and differential forms. Since $C$ is constant, $\left.d(v\rfloor \Lambda_{\alpha}\right)=\frac{\partial v^{i}}{\partial q^{i}} \sqrt{C} \Lambda_{E}=\frac{\partial v^{i}}{\partial q^{i}} \Lambda_{\alpha}$. Hence, Div $v=\frac{\partial v^{i}}{\partial q^{i}}=\operatorname{div} v$.

Statement (iii) follows from (i).

\section{Inclusions with Current Velocities}

Let $\mathbf{v}(t, m)$ be a set-valued vector field and $\boldsymbol{\alpha}(t, m)$ be a set-valued symmetric positive semi-definite $(2,0)$-tensor field on $\mathcal{T}^{n}$. The system of the form

$$
\left\{\begin{array}{l}
D_{S} \xi(t) \in \mathbf{v}(t, \xi(t)), \\
D_{2} \xi(t) \in \boldsymbol{\alpha}(t, \xi(t)) .
\end{array}\right.
$$

is called a first order differential inclusion with current velocities.

Definition 5. We say that (11) on $\mathcal{T}^{n}$ has a solution on $[0, T]$ with initial condition $\xi(0)=\xi_{0}$ if there exists a probability space $(\Omega, \mathcal{F}, \mathrm{P})$ and a process $\xi(t)$ given on $(\Omega, \mathcal{F}, \mathrm{P})$ and taking values in $\mathcal{T}^{n}$ such that $\xi(0)=\xi_{0}$ and for almost all $t \in[0, T]$ inclusion (11) is satisfied P-a.s. by $\xi(t)$.

We suppose that $\mathbf{v}(t, m)$ and $\boldsymbol{\alpha}(t, m)$ satisfy the following conditions:

Assumption 1. Set-valued vector filed $\mathbf{v}(m)$ on $\mathbb{T}^{n}$ is autonomous, upper semi-continuous, uniformly bounded and has closed convex images.

Assumption 2. (i) Set-valued (2,0)-tensor field $\boldsymbol{\alpha}$ on $\mathcal{T}^{n}$ takes values in $S_{L C}$; it is autonomous and upper semi-continuous.

(ii) The values of $\boldsymbol{\alpha}$ are closed and upper semi-continuous.

(iii) For every $m \in \mathcal{T}^{n}$ the set $\mathbf{T}(\boldsymbol{\alpha}(m))$ is convex in $\mathrm{T}_{-}(n)$ and $\mathrm{L}_{\mathrm{C}}(\boldsymbol{\alpha}(m))$ is convex in $\mathrm{L}_{0}(n)$.

Theorem 2. Let the set-valued vector field $\mathbf{v}(t, m)$ on $\mathcal{T}^{n}$ satisfy Assumption 1 and the set-valued (2,0)-tensor field $\boldsymbol{\alpha}(m)$ satisfy Assumption 2. Consider a random element $\xi_{0}$ with values in $\mathcal{T}^{n}$ whose density of distribution with respect to the volume form $\Lambda_{E}$ equals $\sqrt{C} \rho_{0}$ where $\rho_{0}$ is smooth and nowhere equal to zero. Then for initial condition $\xi(0)=\xi_{0}$ inclusion (11) has a solution well defined on the entire interval $t \in[0, T]$.

Proof. First of all, by [7, Theorem 4.11] under the conditions of Assumption 1 for every sequence of positive numbers $\varepsilon_{q} \rightarrow 0$ there exists a sequence of single-valued continuous 
$\varepsilon$-approximations $v_{k}$ of $\mathbf{v}(t, m)$ that point-wise converges to a Borel measurable selector of $\mathbf{v}(t, m)$. Without loss of generality we can suppose those $\varepsilon$-approximations to be smooth.

Since the mappings $T$ and $L_{C}$ are smooth, the set-valued mappings $T \boldsymbol{\alpha}$ with values in $\mathrm{T}_{-}(n)$ and $\mathrm{L}_{C} \boldsymbol{\alpha}$ with values in $\mathrm{L}_{0}(n)$ are upper semicontinuous since such is $\boldsymbol{\alpha}$. By Assumption 2 their values are convex, closed and uniformly bouded. Then by [7, Theorem 4.11] for every sequence of positive numbers $\varepsilon_{q} \rightarrow 0$ there exist sequences of single-valued continuous $\varepsilon_{q}$-approximations that point-wise converge to a Borel measurable selector of T $\boldsymbol{\alpha}$ and $\mathrm{L}_{C} \boldsymbol{\alpha}$, respectively. Take those sequences of approximations as $\varepsilon_{k} \rightarrow 0$ as above. Without loss of generality those approximations can be considered as smooth. Thus, there exists a sequence $\alpha_{k}(m)$ of single-valued smooth and uniformply bounded $(2,0)$-tensor fields from $S_{L C}$ that pointwise converges to a Borel measurable selector $\alpha(m)$ of the setvalued field $\boldsymbol{\alpha}(m)$. We denote the components of $\alpha_{k}(m)$ by $\alpha_{k}^{i j}$.

Construct the sequence of Riemannian metrics $\alpha_{k}(\cdot, \cdot)$ from the tensor fields $\alpha_{k}(m)$ as above.

Consider the sequence of equations

$$
\left\{\begin{array}{c}
D_{S} \xi(t)=v_{k}(\xi(t)) \\
D_{2} \xi(t)=\alpha_{k}(\xi(t))
\end{array} .\right.
$$

Note that by Lemma 2 we can consider the same initial condition $\xi_{0}$ for all those equations since its distribution density with respect to all $\alpha_{k}(\cdot, \cdot)$ coincide. Note also that all $v_{k}$ and $\alpha_{k}$ are uniformly bounded by the same constant since they are $\varepsilon$-approximations of uniformly bounded set-valued mappings. Since all those $\varepsilon$-approximations are at least $C^{1}$-smooth and given on the compact torus, their partial derivatives are uniformly bounded for every $k$ (by a constant depending on $k$ ). Thus all equations (12) satisfy the hypothesis of Theorem 1 , i.e. for every equation there exists a solution. We denote by $\xi_{k}(t)$ the solution of the $k$-th equation.

As it is said in Section 2. every $\alpha_{k}(m)$ can be represented as $\alpha_{k}(m)=A_{k}(m) A_{k}^{*}(m)$, where $A_{k}(m)$ are smooth and uniformly bounded.

On the Banach manifold $C^{0}\left([0, T], \mathcal{T}^{n}\right)$ of continuous curves in $\mathcal{T}^{n}$ we introduce the $\sigma$ algebra $\mathcal{C}$ generated by cylinder sets, and denote by $\mu_{k}$ the measure on $\left(C^{0}\left([0, T], \mathcal{T}^{n}\right), \mathcal{C}\right)$, generated by the solution $\xi_{k}(t)$. We also introduce the family of complete $\sigma$-sub-algebras $\mathcal{P}_{t}$, generated by cylinder sets with bases over $[0, t], t \in[0, T]$, and the family of complete $\sigma$-sub-algebras $\mathcal{N}_{t}$, generated by preimages of Borel sets in $\mathcal{T}^{n}$ under the mappings $x(\cdot) \mapsto$ $x(t)$. It is clear that $\mathcal{N}_{t}$ is a $\sigma$-sub-algebra in $\mathcal{P}_{t}$ and that $\mathcal{P}_{t}$ is the "past" $\sigma$-albera while $\mathcal{N}_{t}$ is the "present" $\sigma$-algebra for coordinate processes on $\left(C^{0}\left([0, T], \mathcal{T}^{n}\right), \mathcal{C}, \mu_{k}\right)$. Recall that a process $\zeta(t)$ that generates measure $\mu_{z}$ eta on $\left(C^{0}\left([0, T], \mathcal{T}^{n}\right), \mathcal{C}\right)$, can be represented as a process on $\left(C^{0}\left([0, T], \mathcal{T}^{n}\right), \mathcal{C}, \mu_{k}\right)$ as the so-called coordinate process $\zeta(t, x(\cdot))=x(t)$.

Lemma 3. The set of measures $\mu_{k}$ on $\left(C^{0}\left([0, T], \mathcal{T}^{n}\right), \mathcal{C}\right)$ is weakly compact.

Proof. Note that since all processes $\xi_{k}(t)$ take values in the compact torus, all $E \xi_{k}(t)$ are uniformly bounded.

Specify two real numbers $0 \leq s<t \leq T$ with small enough difference $t-s$. For every $k$ the increment of $\xi_{k}$ on $[s, t]$ is approximated by the expression $v_{k}\left(\frac{s+t}{2}\right)(t-s)+A_{k}(s)(w(t)-$ $w(s))$. Consider $E\left(\left(v_{k}\left(\frac{s+t}{2}\right)(t-s)+A_{k}(s)(w(t)-w(s))\right)\left(v_{k}\left(\frac{s+t}{2}\right)(t-s)+A_{k}(s)(w(t)-\right.\right.$ $w(s)))^{*}$. Since for all $k$ the norms of $v_{k}$ and $A_{k}$ are bounded by the same constant, one can easily see that among the summands obtained in this expression, only $\alpha_{k}(t-s)$ is an 
infinitesimal of the same order as $t-s$ while all other summands are infinitesimals of an order higher that $t-s$. Thus there exists a constant $h_{1}$ such that if the difference $t-s$ is small enough, the above expression is less than $h_{1}(t-s)$. By integration one can derive from this, that there exists a constant $h>0$, depending on $T$ and on the constant that is a bound of the norms of $v_{k}$ an $\alpha_{k}$, such that for all $0 \leq t_{1}<t_{2} \leq T$ and every $k$ the inequality $E\left(\xi_{k}\left(t_{2}\right)-\xi_{k}\left(t_{1}\right)\right)^{4}<h\left(t_{2}-t_{2}\right)^{2}$ holds. Now the assertion of theorem follows from Lemma [5, Theorem 2 Section 4 Chapter VI].

Let us go on the proof of Theorem 2. Since the set $\left\{\mu_{k}\right\}$ of measures on $\left(C^{0}\left([0, T], \mathcal{T}^{n}\right), \mathcal{C}\right)$ is weakly compact, one can choose a subsequence that weakly converges so a certain measure $\mu$. Without loss of generality we can suppose that the sequence $\mu_{k}$ weakly converges to $\mu$. Consider the coordinate process $\xi(t)$ on the probability space $\left(C^{0}\left([0, T], \mathcal{T}^{n}\right), \mathcal{C}, \mu\right)$, i.e. for every elementary event $x(\cdot) \in C^{0}\left([0, T], \mathcal{T}^{n}\right)$ by definition $\xi(t, x(\cdot))=m(t)$. Recall that $\mathcal{P}_{t}$ is the «past» for $\xi(t)$, while $\mathcal{N}_{t}$ is the «present» for this coordinate process.

By the construction, for every $\xi_{k}(t)$ its quadratic derivative equals $\alpha_{k}\left(\xi_{k}(t)\right)$. This means that for every bounded continuous treal function $f(m(\cdot))$ on $C^{0}\left([0, T], \mathcal{T}^{n}\right)$ that is measurable with respect to $\mathcal{N}_{t}$, the equality

$$
\begin{aligned}
\lim _{\Delta t \rightarrow 0} \int_{C^{0}\left([0, T], \mathcal{T}^{n}\right)}\left[\frac{(m(t+\Delta t)-m(t))(m(t+\Delta t)-m(t))^{*}}{\Delta t}-\right. & \\
& \left.-\alpha_{k}(m(t))\right] f(m(\cdot)) d \mu_{k}=0
\end{aligned}
$$

holds.

Since $\alpha_{k}(t, m)$ pointwise tends to $\alpha(t, m)$ as $k \rightarrow \infty, \alpha_{k}(t, m)$ tends to $\alpha(t, m)$ a.s. with respect to all measures $\mu_{k}$ and with respect to $\mu$. Specify $\delta>0$. By Egorov's theorem (see, e.g., [15]) for every $i$ there exists a subset $\tilde{K}_{\delta}^{i} \subset C^{0}\left([0, T], \mathcal{T}^{n}\right)$ such that $\left(\mu_{i}\right)\left(\tilde{K}_{\delta}^{i}\right)>1-\delta$ and the sequence $\alpha_{k}(m(t))$ on $\tilde{K}_{\delta}^{i}$ converges to $\alpha(m(t))$ uniformly. Introduce $\tilde{K}_{\delta}=\bigcup_{i=0}^{\infty} \tilde{K}_{\delta}^{i}$. The sequence $\alpha_{k}(m(t))$ on $\tilde{K}_{\delta}$ for all $i$ converges to $\alpha(m(t))$ uniformly and $\mu\left(\tilde{K}_{\delta}\right)>1-\delta$.

The field $\alpha(m(t))$ is continuous on a set of complete measure $\mu$ on $C^{0}\left([0, T], \mathcal{T}^{n}\right)$. Indeed, consider the sequence $\delta_{i} \rightarrow 0$ and the corresponding sequence $\tilde{K}_{\delta_{i}}$. By construction, $\alpha(m(t))$ is a unformal limit of the sequence of continuous functions on every $\tilde{K}_{\delta_{i}}$. That is why $\alpha(m(t))$ is continuous on every $\tilde{K}_{\delta_{i}}$, i.e. on any finite union $\bigcup_{i=1}^{n} \tilde{K}_{\delta_{i}}$. Evidently $\lim _{n \rightarrow \infty} \mu\left(\bigcup_{i=1}^{n} \tilde{K}_{\delta_{i}}\right)=1$

Taking into account the uniformal convergence on $\tilde{K}_{\delta}$ for all $k$ (see above) we derive from boundedness of $f(m(\cdot))$ that for $k$ large enough

$$
\left\|\int_{\tilde{K}_{\delta}}\left[\alpha_{k}(m(t))-\alpha(m(t))\right] f(m(\cdot)) d \mu_{k}\right\|<\delta .
$$

Since $f(m(\cdot))$ is bounded, there exists a certain number $\Xi>0$ such that $|f(m(\cdot))|<\Xi$ for all $m(\cdot)$. Recall that all $\alpha_{k}(m)$ and $\alpha(m)$ are uniformly bounded, i.e., their norms are not 
greater than some number $Q>0$. Then, since

$$
\mu_{k}\left(C^{0}\left([0, T], \mathcal{T}^{n}\right) \backslash \tilde{K}_{\delta}\right)<\delta
$$

for all $k$ large enough,

$$
\left\|\int_{C^{0}\left([0, T], \mathcal{T}^{n}\right) \backslash \tilde{K}_{\delta}}\left[\alpha_{k}(m(t))-\alpha(m(t))\right] f(m(\cdot)) d \mu_{k}\right\|<2 \delta Q \Xi
$$

for all $k$ large enough. Since $\delta$ is an arbitrary positive number,

$$
\lim _{k \rightarrow \infty} \int_{C^{0}\left([0, T], \mathcal{T}^{n}\right)}\left[\alpha_{k}(m(t))-\alpha(m(t))\right] f(m(\cdot)) d \mu_{k}=0 .
$$

The function $\alpha(m(t))$ is $\mu$-a.s. continuous and bounded on $C^{0}\left([0, T], \mathcal{T}^{n}\right)$ (see above). Since in addition the measures $\mu_{k}$ weakly converge to $\mu$, by Lemma from [6, section VI.1]

$$
\lim _{k \rightarrow \infty} \int_{C^{0}\left([0, T], \mathcal{T}^{n}\right)} \alpha(m(t)) f(m(\cdot)) d \mu_{k}=\int_{C^{0}\left([0, T], \mathcal{T}^{n}\right)} \alpha(m(t)) f(m(\cdot)) d \mu .
$$

Evidently

$$
\begin{aligned}
\lim _{k \rightarrow \infty} \int_{C^{0}\left([0, T], \mathcal{T}^{n}\right)}\left[\frac{(m(t+\Delta t)-m(t))(m(t+\Delta t)-m(t))^{*}}{\Delta t}\right] f(m(\cdot)) d \mu_{k}= \\
\quad=\int_{C^{0}\left([0, T], \mathcal{T}^{n}\right)}\left[\frac{(m(t+\Delta t)-m(t))(m(t+\Delta t)-m(t))^{*}}{\Delta t}\right] f(m(\cdot)) d \mu .
\end{aligned}
$$

Thus,

$$
\begin{aligned}
\lim _{\Delta t \rightarrow 0} \int_{C^{0}\left([0, T], \mathcal{T}^{n}\right)}\left[\frac{(m(t+\Delta t)-m(t))(m(t+\Delta t)-m(t))^{*}}{\Delta t}\right. & \\
& -\alpha(m(t))] f(m(\cdot)) d \mu=0 .
\end{aligned}
$$

Since $f(m(\cdot))$ is an arbitrary bounded continuous function, measurable with respect to $\mathcal{N}_{t}$, this means that $D_{2} \xi(t)=\alpha(\xi(t))$. But by construction $\alpha(\xi(t)) \in \boldsymbol{\alpha}(\xi(t)) \mu$-a.s.

Next step deals with the current velocity of the solution. By construction $D_{S} \xi_{k}(t)=$ $v_{k}\left(t, \xi_{k}(t)\right)$ for all $k$. This means that for every real bounded continuous function $f$ on $C^{0}\left([0, T], \mathcal{T}^{n}\right)$, measurable with respect to $\mathcal{N}_{t}$, for all $k$ the equality

$$
\lim _{\Delta t \rightarrow 0} \int_{C^{0}\left([0, T], \mathcal{T}^{n}\right)}\left[\frac{m(t+\Delta t)-m(t-\Delta t)}{2 \Delta t}-v_{k}(m(t))\right] f(m(\cdot)) d \mu_{k}=0
$$

holds.

Specify an arbitrary $\varepsilon>0$. Since $\mu_{k}$ weakly converges to $\mu$, there exists $K(\varepsilon)$ such that for $k>K(\varepsilon)$

$$
\begin{gathered}
\| \int_{C^{0}\left([0, T], \mathcal{T}^{n}\right)}\left[\frac{m(t+\Delta t)-m(t-\Delta t)}{2 \Delta t}\right] f(m(\cdot)) d \mu_{k}- \\
-\int_{C^{0}\left([0, T], \mathcal{T}^{n}\right)}\left[\frac{m(t+\Delta t)-m(t-\Delta t)}{2 \Delta t}\right] f(m(\cdot)) d \mu \|<\varepsilon
\end{gathered}
$$


and

$$
\left\|\int_{C^{0}\left([0, T], \mathcal{T}^{n}\right)} f(m(\cdot)) v(m(t)) d \mu_{k}-\int_{C^{0}\left([0, T], \mathcal{T}^{n}\right)} f(m(\cdot)) v(m(t)) d \mu\right\|<\varepsilon .
$$

With the same arguments as above, by the use of Egorov's theorem we prove that

$$
\lim _{k \rightarrow \infty} \int_{C^{0}\left([0, T], \mathcal{T}^{n}\right)}\left[v_{k}(m(t))-v(m(t))\right] f(m(\cdot)) d \mu_{k}=0
$$

and that $v$ is continuous on the set of complete measure. Recall that $v$ is bounded as a selector of bounded set-valued mapping.

Then by Lemma from [6, section VI.1] we obtain

$$
\lim _{k \rightarrow \infty} \int_{C^{0}\left([0, T], \mathcal{T}^{n}\right)} v(m(t)) f(m(\cdot)) d \mu_{k}=\int_{C^{0}\left([0, T], \mathcal{T}^{n}\right)} v(m(t)) f(m(\cdot)) d \mu .
$$

Evidently

$$
\begin{gathered}
\lim _{k \rightarrow \infty} \int_{C^{0}\left([0, T], \mathcal{T}^{n}\right)}\left[\frac{(m(t+\Delta t)-m(t-\Delta t))}{2 \Delta t}\right] f(m(\cdot)) d \mu_{k}= \\
\quad=\int_{C^{0}\left([0, T], \mathcal{T}^{n}\right)}\left[\frac{(m(t+\Delta t)-m(t-\Delta t))}{2 \Delta t}\right] f(m(\cdot)) d \mu .
\end{gathered}
$$

Thus,

$$
\lim _{\Delta t \rightarrow 0} \int_{C^{0}\left([0, T], \mathcal{T}^{n}\right)}\left[\frac{(m(t+\Delta t)-m(t-\Delta t)}{2 \Delta t}-v(m(t))\right] f(m(\cdot)) d \mu=0 .
$$

Since $f(m(\cdot))$ is an arbitrary bounded continuous function, measurable with respect to $\mathcal{N}_{t}$, this means that $D_{S} \xi(t)=v(\xi(t))$. But by construction $v(\xi(t)) \in \mathbf{v}(\xi(t)) \mu$-a.s. This completes the proof.

The research is supported in part by RSF grant No. 14-21-00066 carried out in Voronezh State University.

\section{References}

1. Azarina S.V., Gliklikh Yu.E. Differential inclusions with mean derivatives. Dynamic Systems and Applications, 2007, vol. 16, no. 1, pp. 49-71.

2. Azarina S.V., Gliklikh Yu.E. On Existence of Solutions to Stochastic Differential Equations with Current Velocities. Bulletin of the South Ural State University. Series: Mathematical Modelling, Programming and Computer Software, 2015, vol. 8, no. 4, pp. 100-106. doi: 10.14529/mmp150408

3. Borisovich Yu.G., Gelman B.D, Myshkis A.D, Obukhovskii V.V. [Introduction to the Theory of Set-Valued Mappings and Differential Inclusions]. Moscow, KomKniga Publ., 2005. (in Russian) 
4. Cresson J., Darses S. Stochastic Embedding of Dynamical Systems. Journal of Mathematical Physics, 2007, vol. 48, no. 7, pp. 072703-1-072303-54. doi: $10.1063 / 1.2736519$

5. Gihman I.I., Skorohod A.V. Theory of Stochastic Processes. Vol. 1. New York, Springer, 1974. doi: 10.1007/978-3-642-61943-4

6. Gihman I.I., Skorohod A.V. Theory of Stochastic Processes. V. 3. New York, Springer, 1979. doi: $10.1007 / 978-1-4615-8065-2$

7. Gliklikh Yu.E. Global and Stochastic Analysis with Applications to Mathematical Physics. London, Springer, 2011. doi: 10.1007/978-0-85729-163-9

8. Gliklikh Yu.E., Makarova A.V. On Stochastic Differential Inclusions with Current Velocities. Journal of Computational and Engineering Mathematics, 2015, vol. 2, no. 3, pp. 25-33. doi: 10.14529/jcem150303

9. Makarova A.V. On Solvability of Stochastic Differential Inclusions with Current Velocities II. Global and Stochastic Analysis, 2012, vol. 2. no. 1, pp. 101-112.

10. Nelson E. Derivation of the Schrödinger Equation from Newtonian Mechanics. Physical Review, 1966, vol. 150, no. 4, pp. 1079-1085. doi: 10.1103/PhysRev.150.1079

11. Nelson E. Dynamical Theory of Brownian Motion. Princeton, Princeton University Press, 1967.

12. Nelson E. Quantum fluctuations. Princeton, Princeton University Press, 1985.

13. Parthasarathy K.R. Introduction to Probability and Measure. New York, Springer, 1978.

14. Zhelobenko D.P. Compact Lie Groups and Their Representations. Providence RI, American Mathematical Society, 1973.

15. Yosida K. Functional Analysis. Berlin et. al., Springer - Verlag, 1965.

Yuri E. Gliklikh, Doctor of Physico-Mathematical Sciences, Full Professor, Mathematics Faculty, Voronezh State University (Voronezh, Russian Federation), yeg@math.vsu.ru

Alla V. Makarova, Engineer, Mathematics Faculty, Voronezh State University (Voronezh, Russian Federation), allagm@mail.ru

Received March 1, 2016 
УДК 519.216.2

DOI: $10.14529 /$ jcem160106

\title{
О СТОХАСТИЧЕСКИХ ДИФФЕРЕНЦИАЛЬНЫХ ВКЛЮЧЕНИЯХ С ТЕКУЩИМИ СКОРОСТЯМИ II
}

\author{
Ю. Е. Гликлих, А. В. Макарова
}

Получены теоремы существования решений стохастических дифференциальных включений, заданных в терминах так называемых текущих скоростей (симметрических производных в среднем, прямых аналогов обычных скоростей детерминированных систем) и квадратичных производных в среднем (дающих информацию о коэффициенте диффузии) на плоском $n$-мерном торе. Правые части и для текущей скорости, и для для квадратичной производной многозначны и удовлетворяют некоторым естественным условиям, при которых они имеют $\varepsilon$-аппроксимации, которые поточечно сходятся к измеримым по Борелю селекторам соответствующих многозначных отображений.

Ключевые слова: производные в среднем, текущие скорости, дифференииальные включения.

\section{Литература}

1. Azarina, S.V. Differential inclusions with mean derivatives / S.V. Azarina, Yu.E. Gliklikh // Dynamic Systems and Applications. - 2007. - V. 16, № 1. - P. 49-71.

2. Азарина, С.В. О существовании решений стохастических дифференциальных уравнений с текущими скоростями / С.В. Азарина, Ю.Е. Гликлих // Вестник Южно-Уральского государственного университета. Серия: Математическое моделирование и программирование. - 2015. - Т. 8, № 4. - С. 100-106.

3. Борисович, Ю.Г. Введение в теорию многозначных отображений и дифференциальных включений / Ю.Г. Борисович, Б.Д. Гельман, А.Д. Мышкис, В.В. Обуховский. - М.: Комкнига, 2005.

4. Cresson, J. Stochastic Embedding of Dynamical Systems / J. Cresson, S. Darses // Journal of Mathematical Physics. - 2007. - V. 48, № 7. - P. 072703-1-072303-54.

5. Гихман, И.И. Теория случайных процессов - Т. 1. / И.И. Гихман, А.В. Скороход.М.: Наука, 1971.

6. Гихман, И.И. Теория случайных процессов - Т. 3. / И.И. Гихман, А.В. Скороход. М.: Наука, 1975.

7. Gliklikh, Yu.E. Global and Stochastic Analysis with Applications to Mathematical Physics / Yu.E. Gliklikh. - London: Springer-Verlag, 2011.

8. Gliklikh, Yu.E. On Stochastic Differential Inclusions with Current Velocities Yu.E. Gliklikh, A.V. Makarova // Journal of Computational and Engineering Mathematics. - 2015. - V. 2, № 3. - P. 25-33.

9. Makarova, A.V. On Solvability of Stochastic Differential Inclusions with Current Velocities II / A.V. Makarova // Global and Stochastic Analysis. - 2012. - V. 2, № 1. - P. 101-112. 
10. Nelson, E. Derivation of the Schrödinger Equation from Newtonian Mechanics / E. Nelson // Physical Review. - 1966. - V. 150, № 4. - P. 1079-1085.

11. Nelson, E. Dynamical Theory of Brownian Motion / E. Nelson. - Princeton: Princeton University Press, 1967.

12. Nelson, E. Quantum Fluctuations / E. Nelson. - Princeton: Princeton University Press, 1985.

13. Партасарати, К.Р. Введение в теорию вероятностей и теорию меры / К.Р. Партасарати. - М.: Мир, 1988.

14. Желобенко Д.П. Компактные группы Ли и их представления/ Д.П. Желобенко. М.: Физматлит, 1970.

15. Иосида К. Функциональный анализ / К. Иосида. - М.: Мир, 1967.

Гликлих Юрий Евгенъевич, доктор физико-математических наук, профессор, математический факультет, Воронежский государственный университет (г. Воронеж, Российская Федерачия), уеg@таth.vsu.ru

Макарова Алла Викторовна, инженер, математический факультет, Воронежский государственный университет (2. Воронеж, Российская Федерачия), allagm@mail.ru

Поступила в редакиию 1 марта 2016 г. 